\title{
Discrimination of malignant transformation from benign endometriosis using a near-infrared approach
}

\author{
NAOKI KAWAHARA ${ }^{1}$, YUKI YAMADA ${ }^{1}$, FUMINORI ITO $^{1}$, WATARU HOJO ${ }^{2}$, \\ TAKUYA IWABUCHI $^{2}$ and HIROSHI KOBAYASHI ${ }^{1}$ \\ ${ }^{1}$ Department of Obstetrics and Gynecology, Nara Medical University, Kashihara, Nara 634-8522; \\ ${ }^{2}$ Department of Research and Development, Cellspect Co., Ltd., Morioka, Iwate 020-0857, Japan
}

Received September 20, 2017; Accepted January 16, 2018

DOI: $10.3892 /$ etm.2018.5779

\begin{abstract}
The aim of the present single-center retrospective study was to investigate the discrimination of malignant transformation from ovarian endometrioma (OE) using a near-infrared approach ex vivo. Cystic fluid samples were collected from patients with OE $(n=34)$ and endometriosis-associated ovarian cancer $($ EAOC) $(n=12)$. The light reflected from each sample of cystic fluid [change in luminance, $\Delta \mathrm{l}\left(\mathrm{cd} / \mathrm{m}^{2}\right)=$ background luminance-cystic fluid luminance at $800 \mathrm{~nm}$ ] was spectrally measured by a near-infrared CCD camera with band-path filter $(800 \mathrm{~nm})$. The $\Delta \mathrm{l}$ in EAOC was significantly lower compared with that in OE. On regression analysis, a positive correlation was observed between the $\Delta \mathrm{l}$ and $\mathrm{Hb}$ level in the cystic fluid, and this association was exponential. The diagnostic sensitivity and specificity of $\Delta \mathrm{l}$ was 83.3 and $94.1 \%$ at the cutoff value of $21.5 \mathrm{~cd} / \mathrm{m}^{2}$, with an area under the ROC curve of 0.897 . The present ex vivo study potentially provides a powerful near-infrared approach for quantitative discrimination between EAOC and benign OE, with high sensitivity and specificity, which may have clinical applications.
\end{abstract}

\section{Introduction}

Ovarian cancer represents one of the leading causes of cancer-related mortality, with an increasing prevalence in Japan (1). The pathogenesis of ovarian cancer is complex, and is affected by numerous epigenetic and genetic factors (2). Endometriosis is usually a benign disorder, but is associated with an increased risk for developing ovarian (3) and endometrial cancers (4). Endometrioid and clear cell carcinoma of

Correspondence to: Professor Hiroshi Kobayashi, Department of Obstetrics and Gynecology, Nara Medical University, 840 Shijo-cho, Kashihara, Nara 634-8522, Japan

E-mail: hirokoba@naramed-u.ac.jp

Key words: near infrared, endometriosis, malignant transformation, ovarian cancer the ovary (endometriosis-associated ovarian cancer, EAOC) originates from endometriosis.

Magnetic resonance (MR) imaging may be used as an adjunctive method to distinguish EAOC from benign ovarian endometrioma (OE). Specifically, we have recently demonstrated that the MR transverse relaxation rate provides a noninvasive predictive tool to discriminate between EAOC and OE (5). However, the implementation of MR imaging in the outpatient clinic is often difficult.

Recent progress in research on the pathogenesis of EAOC is based on key developments in two areas: i) New mechanistic concepts regarding the pathogenesis of EAOC revealed a key role of hemoglobin ( $\mathrm{Hb})$, heme and iron-induced oxidative stress in the OE cystic fluid, wherein an imbalance between the overproduction of iron-induced oxidative stress and defense mechanisms could trigger DNA damage and carcinogenesis; and ii) the establishment of novel approaches to identify stress biomarkers, such as $\mathrm{Hb}$ and iron, for the prediction of malignant transformation $(2,6,7)$. A recent ex vivo study revealed that electronic absorption spectroscopy using visible light at 580 and $620 \mathrm{~nm}$ provides a measure of $\mathrm{Hb}$ species in endometriotic cystic fluid by monitoring the relative concentrations of oxyhemoglobin and methemoglobin, respectively (8). The $620 / 580 \mathrm{~nm}$ peak ratio of cystic fluid in EAOC patients was significantly lower compared with that measured in women with benign OE (8). Therefore, the cystic fluid $\mathrm{Hb}$ species may be used as biomarkers in the differential diagnosis between EAOC and OE. However, one limitation of this absorption-based method is that the camera must be placed opposite a halogen white light source, which is disadvantageous as light in the visible spectrum does not penetrate tissue.

Recent advances in optical technology have led to innovative quantitative monitoring tools, which include spatially-resolved reflectance, diffuse optical spectroscopy, diffuse optical tomography and diffuse correlation spectroscopy $(9,10)$. Optical spectroscopy and tomography utilize the near-infrared spectral region to provide quantitative determination of several important biological chromophores, such as $\mathrm{Hb}$ (11) or cytochrome $c$ oxidase (12). Furthermore, light in the near-infrared spectrum efficiently penetrates tissue, including bone and muscle (11). When near-infrared light is shone through cystic fluid, the influence of photon 
attenuation and scattering varies depending on $\mathrm{Hb}$ concentration. A backscattered photon or an on-axis luminance measurement can be detected by means of appropriate optical apparatus. We hypothesized that the changes in luminance across a fluid can typically be used to determine the $\mathrm{Hb}$ concentration of the cystic fluid using a near-infrared sensor camera. Thus, we developed a rapid and sensitive ex vivo assay based on the changes of dynamic light scattering or changes in luminance across the cystic fluid. Furthermore, we investigated the potential of the luminance measurement as an objective optical method to discriminate EAOC from benign $\mathrm{OE}$.

\section{Materials and methods}

Study population. The research protocol was approved by the Nara Medical University Review Boards, and written informed consent was obtained from all subjects. A total of 46 patients with OE $(n=34)$ or EAOC $(n=12)$ were recruited between February 2013 and January 2015 at the Department of Gynecology, Nara Medical University Hospital (Kashihara, Japan). Histopathological examination confirmed the diagnoses of benign $\mathrm{OE}$ and EAOC. All cystic fluid samples were collected from the patients during surgery, and an aliquot of each sample was stored at $-80^{\circ} \mathrm{C}$ until testing.

Instrumentation and system design. Narrow-band optical filtering is required to achieve the highest signal-to-noise ratio (SNR) as an optical enhancement technology. During our preliminary study, the SNR was determined analytically using a band-path filter with varying wavelengths (750-1,000 nm), and the results were validated experimentally. We found a single optimum for the optical path length of the filter at $800 \mathrm{~nm}$ (data not shown). This was applied to in the current system.

The instrument was designed and set up. A diagram of the light path is illustrated in Fig. 1. This figure indicates the luminance (light reflected from the sample) measurement of the cystic fluid sample. Measurements were obtained by performing ex vivo phantom experiments. An aliquot of the cystic fluid sample $(1 \mathrm{ml})$ was transferred to a disposable cuvette (10-mm wide x $10-\mathrm{mm}$ thick). The light source was a halogen lamp with a $300 \mathrm{~W}$ quartz-tungsten-halogen bulb (EXR 82v; Eiko, Co., Ltd., Hitachinaka, Japan). The distance between the halogen light and the cystic fluid was $50 \mathrm{~mm}$. Halogen light illuminated the sample and a near-infrared CCD camera with band-path filter $(800 \mathrm{~nm})$ recorded the light signal reflected from the sample. The change in luminance $\left[\Delta 1\right.$ value $\left.\left(\mathrm{cd} / \mathrm{m}^{2}\right)\right]$ was calculated by subtracting a sample blank for each specimen $(\Delta \mathrm{l}=$ background luminance - cystic fluid luminance at $800 \mathrm{~nm})$.

$\mathrm{Hb}$ assay. Cystic fluid total $\mathrm{Hb}$ concentrations were measured as described previously $(6,8,13)$. From the correlation data, a formula was calculated to convert heme levels (mg/l) to hemoglobin $(\mathrm{g} / \mathrm{dl})$. We then investigated the correlation between $\Delta \mathrm{l}$ and $\mathrm{Hb}$ in cystic fluid.

Effects of an anatomical barrier on surface reflectance. Transvaginal ultrasound imaging is replacing radiological methods in the investigation of ovarian tumors. Due to the presence of an anatomical barrier (which may include an ovarian cyst wall or vaginal connective/muscle tissue) in this type of imaging, ex vivo experiments using appropriate modeling are important to establish a clinically relevant model. To investigate the effect of such an anatomical barrier, the surface of a disposable cuvette was covered with pieces of commercial Japanese chicken of different thicknesses ( 5 and $10 \mathrm{~mm}$ ). The $\Delta \mathrm{l}$ value was measured by recording the light scattered (surface luminance) from the cystic fluid that was covered by these barriers. We generated three sets of experiments: Experiment $1(0 \mathrm{~mm}$; the surface of the cuvette was not covered); experiment $2(5 \mathrm{~mm}$; the surface of the cuvette was covered by a 5 -mm-thick piece of chicken); and experiment 3 (10 $\mathrm{mm}$; the surface of the cuvette was covered by a $10-\mathrm{mm}$-thick piece of chicken).

Statistical analysis. Statistical analysis was conducted using the SPSS 22.0 software package (IBM Corp., Armonk, NY, USA). Comparisons of non-parametric data ( $\Delta \mathrm{l}$ and $\mathrm{Hb}$ levels) between the $\mathrm{OE}$ and EAOC groups were performed using the Mann-Whitney U test. Correlation analysis was performed using Pearson's correlation coefficient. The optimal cutoff value was defined according to analysis of the receiver operating characteristic (ROC) curve. The sensitivity and specificity of detection were calculated on the basis of cutoff value to differentiate EAOC from benign $\mathrm{OE}$. The area under the ROC curve (AUC) was also calculated for each marker. $\mathrm{P}<0.05$ was considered to indicate a statistically significant difference.

\section{Results}

$\Delta l$ and $H b$ of cystic fluid samples. The clinical characteristics, cystic fluid $\Delta \mathrm{l}$ levels and $\mathrm{Hb}$ concentrations of patients are summarized in Table I. Subjects in the EAOC group were older compared with the OE group $(\mathrm{P}<0.001)$. Fig. 2 shows box and whisker plots representing the median level and interquartile range (box) of $\Delta \mathrm{l}$ and $\mathrm{Hb}$ for each studied group. The EAOC patients showed significantly lower $\Delta \mathrm{l}$ values compared with the $\mathrm{OE}$ group $(\mathrm{P}<0.001)$ (Table I; Fig. 2A). The cystic fluid levels of $\mathrm{Hb}$ were also significantly lower in EAOC patients compared with OE patients (Table I; Fig. 2B). These results indicated that the $\mathrm{OE}$ and EAOC groups were clearly separated.

ROC curve in EAOC group vs. benign OE group. The sensitivity and specificity of cystic fluid $\Delta \mathrm{l}$ level for the diagnosis of malignant transformation were 83.3 and $94.1 \%$, respectively, using a cutoff value of 21. The AUC was 0.897 (Fig. 3A; Table II, experiment 1). A Hb level of $1.99 \mathrm{~g} / \mathrm{dl}$ was identified to detect EAOC with a sensitivity of $100 \%$ and a specificity of $91.7 \%$, and an AUC of 0.988 (Fig. 3B). Since the patients with EAOC were significantly older than those with OE, correlations between age and each parameter were evaluated using Pearson's correlation coefficient. The age distribution of the subjects is shown in Fig. 4. There were no significant correlations between age and cystic fluid $\Delta \mathrm{l}$ (Fig. 4A; $\mathrm{r}=-0.128, \mathrm{P}=0.470$ ) or $\mathrm{Hb}$ level (Fig. $4 \mathrm{~B} ; \mathrm{r}=-0.159, \mathrm{P}=0.370$ ) in the OE group. There were also no correlations between age 


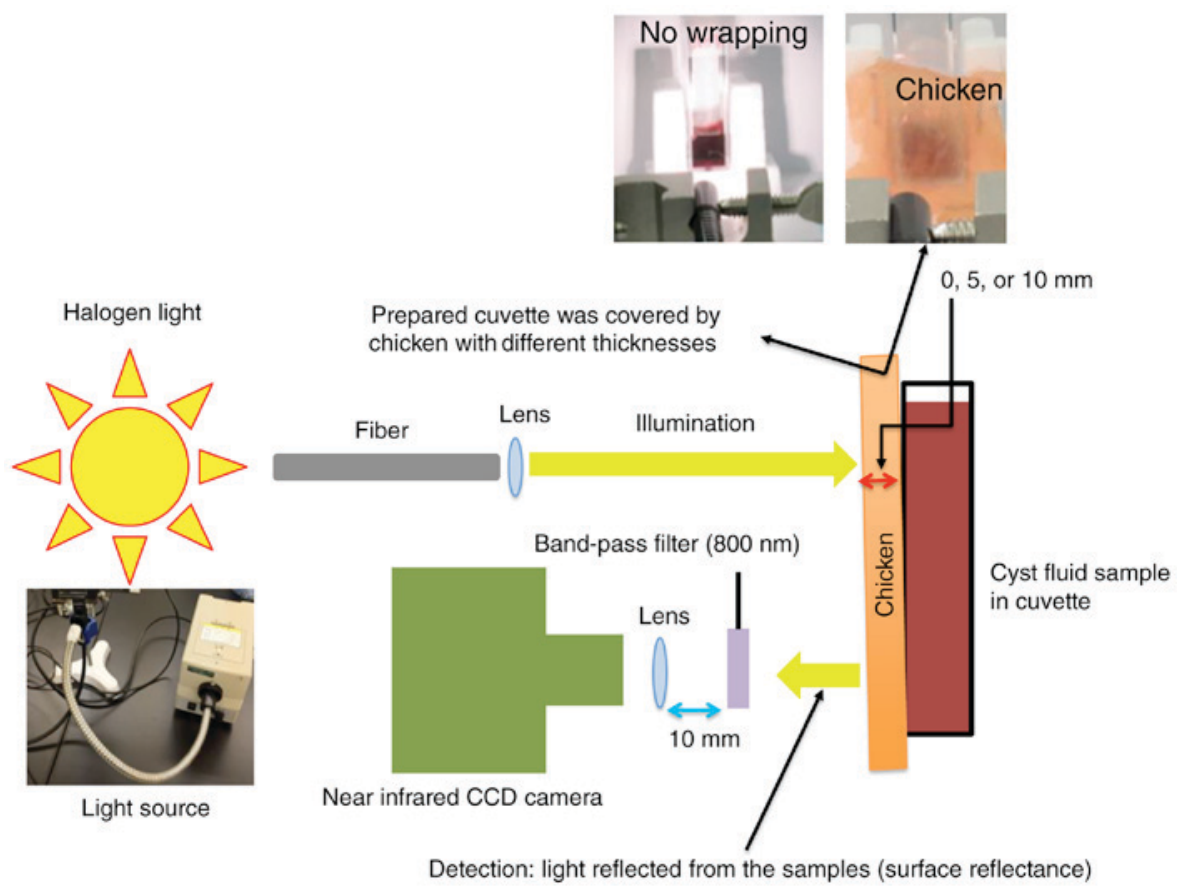

Figure 1. Schematic illustration of experimental setup to acquire a light reflected from the samples. Schematic diagram of the optical arrangement used for luminance measurements. The experimental arrangement of the halogen light illumination and photodetector are illustrated. The light source and CCD camera are precisely aligned to image the light signal. The prepared cuvette was covered by a commercially available chicken with a different thickness $(0,5 \mathrm{and} 10 \mathrm{~mm})$.

Table I. Patient demographics and tumor characteristics of two groups.

Patient and

clinical characteristics

OE

EAOC

P-value

Number

Age (years)

Median (range)

Mean \pm SD

Cyst size $(\mathrm{cm})^{\mathrm{a}}$

Median (range)

Mean \pm SD

FIGO stage

Pathology
34

$39.0(26-51)$

$38 \pm 7$

$7.0(2.7-19.3)$

$7.7 \pm 3.2$

Endometriosis
12

$49.5(36-69)$

$49 \pm 11$

$11.0(4.2-22.5)$

$12.1 \pm 5.7$

Ia $(n=5)$, Ib $(n=1)$, Ic $(n=6)$

Clear cell carcinoma $(n=6)$

Endometrioid carcinoma $(n=3)$

Mucinous carcinoma $(\mathrm{n}=1)$

Serous carcinoma $(n=1)$

Seromucinous carcinoma $(n=1)$

$\Delta \mathrm{l}\left(\mathrm{cd} / \mathrm{m}^{2}\right)$

$\begin{array}{lc}\text { Median (range) } & 29.0(18.2-41.4) \\ \text { Mean } \pm \text { SD } & 29.6 \pm 5.0\end{array}$

$\mathrm{Hb}(\mathrm{g} / \mathrm{dl})$

Median (range)

$6.1(2.2-42.8)$

$9.5 \pm 9.3$

${ }^{a}$ Maximum diameter of tumor cysts. OE, ovarian endometrioma; EAOC, endometriosis-associated ovarian cancer; FIGO, International Federation of Gynecology and Obstetrics; SD, standard deviation.

and cystic fluid $\Delta \mathrm{l}$ (Fig. $4 \mathrm{~A} ; \mathrm{r}=0.518, \mathrm{P}=0.084)$ or $\mathrm{Hb}$ level (Fig. 4B; $r=0.532, \mathrm{P}=0.075$ ) in the EAOC group. Fig. 5 shows a scatter plot of correlation between $\Delta \mathrm{l}$ and cystic fluid $\mathrm{Hb}$ level for the OE and the EAOC groups. When the interaction 
Table II. Effects of an anatomical barrier against surface reflectance.

\begin{tabular}{|c|c|c|c|c|c|c|c|c|}
\hline Patients & $\begin{array}{c}\mathrm{OE} \\
(\mathrm{n}=34)\end{array}$ & $\begin{array}{l}\text { EAOC } \\
(n=12)\end{array}$ & AUC & $95 \% \mathrm{CI}$ & P-value & Cut-off & $\begin{array}{l}\text { Sensitivity } \\
(\%)\end{array}$ & $\begin{array}{cc}\text { Specificity } & \text { PPV } \\
(\%) & (\%)\end{array}$ \\
\hline
\end{tabular}

Experiment 1

(Samples

not-covered

by a chicken)

$\Delta \mathrm{l}\left(\mathrm{cd} / \mathrm{m}^{2}\right)$

$\begin{array}{cc}29.6 \pm 5.0 & 14.4 \pm 10.8 \\ (18.2-41.4) & (-5.5-32.3)\end{array}$

$0.897 \quad 0.772-1.000<0.001$

21.5

83.3

94.1

$83.3 \quad 94.1$

Experiment 2

(Samples covered

by a $5 \mathrm{~mm}$-thick

chicken)

$\Delta \mathrm{l}\left(\mathrm{cd} / \mathrm{m}^{2}\right)$

Experiment 3

(Samples covered by a $10 \mathrm{~mm}$-thick chicken)

$\Delta \mathrm{l}\left(\mathrm{cd} / \mathrm{m}^{2}\right)$

$$
\begin{array}{ll}
-9.9 \pm 5.1 & -17.6 \pm 7.6 \\
(-20.1-2.0) & (-26.3-6.3)
\end{array}
$$

$\begin{array}{llll}0.778 & 0.609-0.948 & 0.005 & -15.5\end{array}$

66.7

85.3

$61.5 \quad 87.9$

Upper, experiment 1; middle, experiment 2; and lower, experiment 3 panels represent the $\Delta \mathrm{l}$ values and the discriminative value of each parameter in the OE and EAOC groups. OE, ovarian endometrioma; EAOC, endometriosis-associated ovarian cancer; AUC, area under the curve; PPV, positive predictive value; NPV, negative predictive value.

A

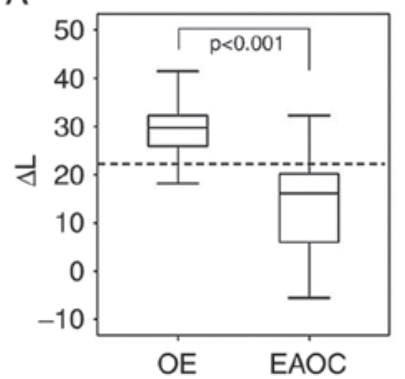

B

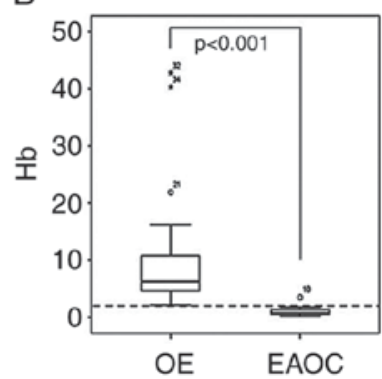

Figure 2. (A) Cystic fluid $\Delta \mathrm{l}$ and (B) $\mathrm{Hb}$ levels in patients with EAOC and $\mathrm{OE}$. This image shows the distribution of marker levels for each studied group. Cystic fluid levels were studied in patients with EAOC $(n=12)$ and OE $(n=34)$. (A) The EAOC patients showed significantly lower $\Delta \mathrm{l}$ values compared with the OE group $(\mathrm{P}<0.001)$. (B) The EAOC patients showed significantly lower $\mathrm{Hg}$ values compared with the $\mathrm{OE}$ group $(\mathrm{P}<0.001)$.

between $\Delta \mathrm{l}$ and $\mathrm{Hb}$ was analyzed using a linear model, the best model fitted an exponential function. Therefore, data were linearized by log transformation. The association of $\Delta \mathrm{l}$ with $\mathrm{Hb}$ became steeper with lower Hb levels ( $<2 \mathrm{~g} / \mathrm{dl}) . \Delta \mathrm{l}$ was strongly correlated with the cystic fluid $\mathrm{Hb}$ concentration $(\mathrm{r}=0.558, \mathrm{P}<0.001)$.

Effects of an anatomical barrier on surface reflectance. The results of the ex vivo approach are summarized in Table II, which shows the comparison of measurements

A

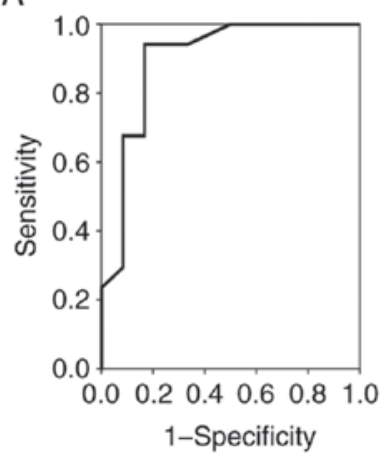

B

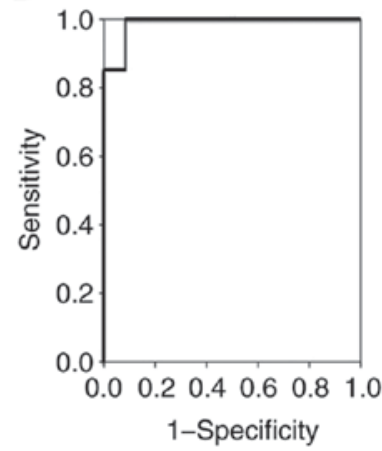

Figure 3. ROC curve comparing (A) $\Delta \mathrm{l}$ and (B) $\mathrm{Hb}$ levels in patients with EAOC group vs. patients with benign OE group. Receiver-operating characteristic (ROC) analysis shows a higher area under the curve (AUC) for ratio of the pixel density value of $\Delta \mathrm{l}$ and $\mathrm{Hb}$. (A) The proper cut off for the $\Delta \mathrm{l}$ level which was determined for the diagnosis of malignant transformation in our study was 21.5 . The area under the ROC curve of the $\Delta 1$ level was 0.897 . The ROC curve exhibited $83.3 \%$ sensitivity and $94.1 \%$ specificity. (B) The best cut-off point for cystic fluid $\mathrm{Hb}$ level was $1.99 \mathrm{mg} / \mathrm{l}$. The area under the ROC curve was 0.988 . Sensitivity and specificity for detecting EAOC from benign OE were 100 and $91.7 \%$, respectively.

obtained from each experiment. The AUC for diagnosing EAOC from OE was 0.897 (experiment 1: Sensitivity, $83.3 \%$; specificity, 94.1\%), 0.859 (experiment 2: Sensitivity, $75.0 \%$; specificity, 91.2\%) and 0.778 (experiment 3: Sensitivity, 66.7\%; specificity, $85.3 \%$ ). 
A

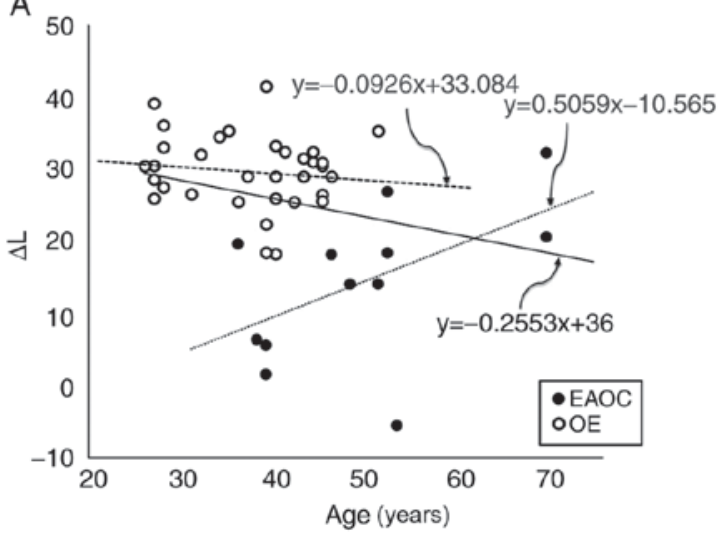

B

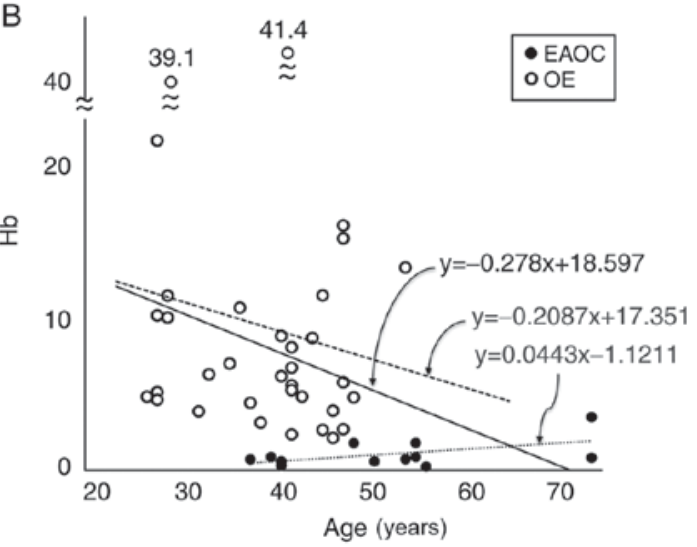

Figure 4. Cystic fluid (A) $\Delta \mathrm{l}$ and (B) Hb levels per age for subjects in the $\mathrm{OE}$ and EAOC samples. Open circle represents an individual value of $\mathrm{OE}$ subject. Closed circle represents an individual value of EAOC subject. (A) Correlation between cystic fluid $\Delta \mathrm{l}$ levels and age at surgery in women with $\mathrm{OE}(\mathrm{y}=-0.0926 \mathrm{x}+33.084, \mathrm{r}=-0.128, \mathrm{P}=0.470)$ and in patient with EAOC $(y=0.5059 x-10.565, r=0.518, P=0.084) .$,$x , age at surgery; y$, cystic fluid $\Delta 1$ level $\left(\mathrm{cd} / \mathrm{m}^{2}\right)$. (B) Correlation between cystic fluid $\mathrm{Hb}$ levels and age at surgery in women with $\mathrm{OE}(\mathrm{y}=-0.2087 \mathrm{x}+17.351, \mathrm{r}=-0.159, \mathrm{P}=0.370)$ and in patient with EAOC $(\mathrm{y}=0.0443 \mathrm{x}-1.1211, \mathrm{r}=0.532, \mathrm{P}=0.075$,). $\mathrm{x}$, age at surgery; $\mathrm{y}$, cystic fluid $\mathrm{Hb}$ level (mg/l).

\section{Discussion}

To the best of our knowledge, this is the first ex vivo study of cystic fluid measurements via optical properties at $800 \mathrm{~nm}$, which can discriminate malignant transformation from benign $\mathrm{OE}$. The $\Delta \mathrm{l}$ of cystic fluid from the EAOC group was significantly lower compared with that of the OE group $(\mathrm{P}<0.001)$. $\Delta \mathrm{l}$ level could serve as a simple, rapid and accurate method to discriminate EAOC from benign $\mathrm{OE}$, with high sensitivity (83.3\%) and specificity (94.1\%). In our ex vivo experiments, the samples were covered by 5 - or $10 \mathrm{~mm}$-thick pieces of chicken. Our measurements showed that the $10 \mathrm{~mm}$-thick sample attenuated the power of $\Delta \mathrm{L}$ to discriminate between benign and malignant specimens, with relatively lower sensitivity $(66.7 \%)$ and specificity (85.3\%).

Furthermore, the cystic fluid $\mathrm{Hb}$ concentrations were reduced in patients with EAOC (6-8). The $\Delta \mathrm{l}$ values and total $\mathrm{Hb}$ concentrations in 46 samples exhibited an exponential correlation ( $\mathrm{r}=0.558)$, suggesting that the $\Delta \mathrm{l}$ value may reflect the $\mathrm{Hb}$ concentration. The present results were in agreement with those of Yoshimoto et al (6), who reported the cystic fluid concentration of total iron, heme iron, free iron

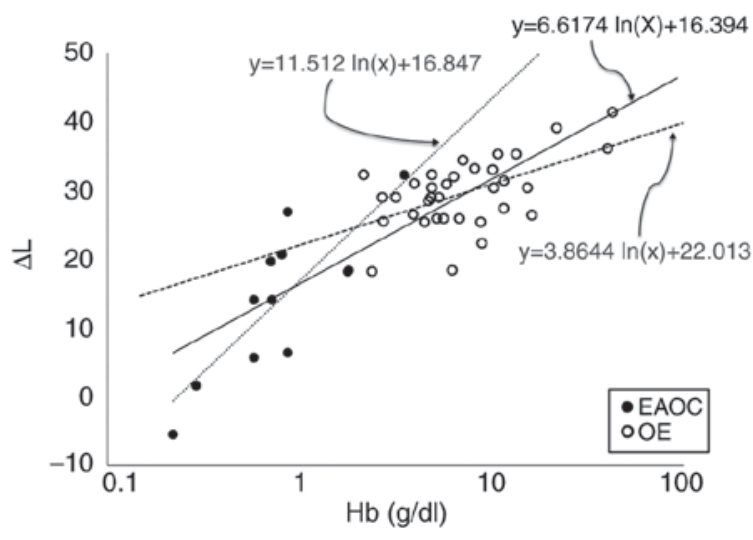

Figure 5. Relation between $\Delta \mathrm{l}$ and $\mathrm{Hb}$ in the $\mathrm{OE}$ group and the EAOC group. The relationship between $\Delta \mathrm{l}$ and $\mathrm{Hb}$ level was better described by an exponential function $(\mathrm{y}=6.6174 \ln (\mathrm{x})+16.394, \mathrm{r}=0.558, \mathrm{P}<0.001)$. Correlation between cystic fluid $\Delta \mathrm{l}$ levels and cystic fluid $\mathrm{Hb}$ level in women with $\mathrm{OE}$ $(\mathrm{y}=3.8644 \ln (\mathrm{x})+22.013, \mathrm{r}=0.585, \mathrm{P}<0.001)$ and in patient with EAOC $(\mathrm{y}=11.512 \ln (\mathrm{x})+16.847, \mathrm{r}=0.704, \mathrm{P}=0.011) . \mathrm{x}$, cystic fluid Hb level (mg/l); y, cystic fluid $\Delta 1$ level $\left(\mathrm{cd} / \mathrm{m}^{2}\right)$.

and $\mathrm{Hb}$ species (6,7). Previous studies of $\mathrm{Hb}$ species have reported differences between OE and EAOC samples (8). Transvaginal ultrasound-guided luminance measurements using near-infrared approaches may advance medical imaging technology as a tool for discriminating malignant transformation in endometriosis.

Despite the advantages discussed above, there are several limitations in the present study. Firstly, an exponential curve of the $\mathrm{Hb}$ levels was a better-fitting model compared with the linear model. However, whether and how the $\Delta \mathrm{l}$ level reflects absolute $\mathrm{Hb}$ concentration has not yet been studied. We could not exclude the possibility of cross-contamination of other factors, such as heme iron and free iron, in these data acquired at an 800-nm wavelength. Secondly, a major limitation is the lack of large-scale evaluation. Finally, the complexity of reproductive organ anatomy poses several challenges for in vivo luminance imaging. Non-invasive imaging in deep tissue requires a near-infrared $\mathrm{CCD}$ camera with strong sensitivity and high spatial resolution. By adding detectors at multiple distances from the emitted light source, specific algorithms can subtract superficial light absorption from deep absorption to provide qualitative information of the cystic fluid $\mathrm{Hb}$ level (11). Despite these limitations, there is a great need to develop a clinically useful, noninvasive and reliable tool that accurately predicts the malignant transformation of endometriosis.

In conclusion, the luminance value obtained from ex vivo cystic fluid samples at an $800-\mathrm{nm}$ wavelength may discriminate EAOC from benign OE patients. Transvaginal near-infrared approaches may provide a non-invasive assessment of malignant transformation of $\mathrm{OE}$, and may have further clinical applications in an outpatient setting.

The aim of this study was to investigate the discrimination of malignant transformation from OE using a near-infrared approach ex vivo. The diagnostic sensitivity and specificity for $\Delta \mathrm{l}$ (change in luminance, $\mathrm{cd} / \mathrm{m}^{2}$ ) were 83.3 and $94.1 \%$, respectively, at the cutoff value of $21.5 \mathrm{~cd} / \mathrm{m}^{2}$, with an AUC of 0.897 . This ex vivo study potentially provides a powerful near-infrared approach for discrimination between 
EAOC and benign OE, with high sensitivity and specificity. This study provides a basis for developing future clinical approaches.

\section{Acknowledgements}

This study was supported by grant-in-aid for Scientific Research from the Ministry of Education, Science, and Culture of Japan (H.K.).

\section{References}

1. Ushijima K: Current status of gynecologic cancer in Japan. J Gynecol Oncol 20: 67-71, 2009.

2. Koshiyama M, Matsumura N and Konishi I: Recent concepts of ovarian carcinogenesis: Type I and type II. Biomed Res Int 2014: 934261, 2014.

3. Brinton LA, Gridley G, Persson I, Baron J and Bergqvist A: Cancer risk after a hospital discharge diagnosis of endometriosis. Am J Obstet Gynecol 176: 572-579, 1997.

4. Yu HC, Lin CY, Chang WC, Shen BJ, Chang WP and Chuang CM; Task Force on Carcinogenesis of Endometrial Cancer: Increased association between endometriosis and endometrial cancer: A nationwide population-based retrospective cohort study. Int J Gynecol Cancer 25: 447-452, 2015.

5. Yoshimoto C, Takahama J, Iwabuchi T, Uchikoshi M, Shigetomi $\mathrm{H}$ and Kobayashi $\mathrm{H}$ : Transverse relaxation rate of cyst fluid can predict malignant transformation of ovarian endometriosis. Magn Reson Med Sci 16: 137-145, 2017.
6. Yoshimoto C, Iwabuchi T, Shigetomi H and Kobayashi H: Cyst fluid iron-related compounds as useful markers to distinguish malignant transformation from benign endometriotic cysts. Cancer Biomark 15: 493-499, 2015.

7. Iwabuchi T, Yoshimoto C, Shigetomi $\mathrm{H}$ and Kobayashi H: Oxidative stress and antioxidant defense in endometriosis and its malignant transformation. Oxid Med Cell Longev 2015: 848595, 2015.

8. Iwabuchi T, Yoshimoto C, Shigetomi H and Kobayashi H: Cyst fluid hemoglobin species in endometriosis and its malignant transformation: The role of metallobiology. Oncol Lett 11: 3384-3388, 2016.

9. Cuccia DJ, Abookasis D, Frostig RD and Tromberg BJ: Quantitative in vivo imaging of tissue absorption, scattering, and hemoglobin concentration in rat cortex using spatially modulated structured light. In: In vivo optical imaging of brain function. Frostig RD (ed). 2nd edition. Boca Raton (FL). CRC Press/Taylor \& Francis, 2009.

10. Wilson JR, Mancini DM, McCully K, Ferraro N, Lanoce V and Chance B: Noninvasive detection of skeletal muscle underperfusion with near-infrared spectroscopy in patients with heart failure. Circulation 80: 1668-1674, 1989.

11. Steppan J and Hogue CW Jr: Cerebral and tissue oximetry. Best Pract Res Clin Anaesthesiol 28: 429-439, 2014

12. Kolyva C, Ghosh A, Tachtsidis I, Highton D, Smith M and Elwell CE: Dependence on NIRS source-detector spacing of cytochrome c oxidase response to hypoxia and hypercapnia in the adult brain. Adv Exp Med Biol 789: 353-359, 2013.

13. Kim YJ, Kim S, Kim JW and Yoon G: Data preprocessing and partial least squares regression analysis for reagentless determination of hemoglobin concentrations using conventional and total transmission spectroscopy. J Biomed Opt 6: 177-182, 2001. 